\title{
Frames als Mittel zur systematischen Klassifikation von psychischen Störungen
}

\author{
Gottfried Vosgerau, Jürgen Zielasek \& Patrice Soom
}

\begin{abstract}
Der vorliegende Beitrag hat zum Ziel, anhand eines Beispiels die Vorteile der Frame-Darstellung psychischer Störungen zu verdeutlichen. Diese Vorteile liegen in erster Linie darin, eine systematische Beschreibung verschiedener Faktoren und deren Interaktionen zu erlauben. Da verschiedene Beschreibungsebenen in Frames integriert werden können, können somit auch komplexe Zusammenhänge zwischen verschiedenen psychischen Funktionsbereichen sowie eventuell „diffuse“ Auswirkungen einzelner Störungen auf unterschiedlichste psychische Bereiche erfasst werden. Nachdem im ersten Abschnitt zunächst einige Grundüberlegungen zur Klassifikation von psychischen Störungen vorgestellt werden, wird der Beitrag anhand des Beispiels des Phänomens der Fremdbeeinflussung, wie es bei Schizophrenie auftritt, schrittweise einen komplexen Frame erstellen. Hierzu werden zunächst Frames allgemein charakterisiert, um dann schrittweise über die Diskussion des Phänomens einen komplexen Frame zu erstellen. Die spezifischen Vorteile der Frame-Darstellung werden parallel zur Erstellung des Frames diskutiert und am Ende des Beitrages in der Zusammenfassung gebündelt dargestellt.
\end{abstract}

\section{Stand und Desiderata der Klassifikation psychischer Störungen}

Aktuell werden für die Klassifikation psychischer Störungen international vor allem zwei Klassifikationssysteme angewendet: zum einen das Diagnostic and Statistical Manual of Mental Disorders der American Psychiatric Association (DSM-5, American Psychiatric Association 2013) und das Kapitel „Psychische und 
Verhaltensstörungen“ in der Internationalen statistischen Klassifikation der Krankheiten und verwandter Gesundheitsprobleme der Weltgesundheitsorganisation (ICD-10, World Health Organization 1992). Während in der Forschung und in den USA auch im klinischen Alltag die Nutzung von DSM-5 dominiert, wird in den meisten europäischen Ländern und auch in Deutschland zur klinischen Diagnose und zur Abrechnung mit den Kassen nach gesetzlicher Vorschrift die ICD-10 verwendet (Gaebel/Zielasek 2011). Beide Kataloge werden regelmäßig überarbeitet und neu aufgelegt. So ist die fünfte Version des DSM im Mai 2013 erschienen, die elfte Version der ICD ist gegenwärtig in Arbeit und wird wahrscheinlich 2017 erscheinen.

Obwohl sich die Details der Klassifikation in den beiden Werken unterscheiden (z. B. in der Mindestsymptomdauer bei der Schizophrenie), sind der Aufbau und die Systematik durchaus vergleichbar für die hier zu diskutierenden Punkte. Mit ICD-11 dürfte die Annäherung der beiden Klassifikationssysteme weitere Fortschritte machen, z. B. im Bereich der psychotischen Störungen, wobei jedoch auch einige Unterschiede bestehen bleiben dürften (Gaebel/Zielasek/Cleveland 2012). Weiterhin gilt, dass die beiden Klassifikationssysteme nicht nur der Wissenschaft dienen, sondern auch klinisch-praktischen Anforderungen genügen müssen: So muss die Definition einer Krankheit etwa derart sein, dass die Diagnostik im klinischen Alltag auch mit vertretbarem Aufwand durchgeführt werden kann. Weiterhin bilden die Kataloge die Grundlage von rechtlichen Entscheidungen, und zwar nicht nur vor Gericht, sondern auch in Bezug auf das Recht auf eine von der Krankenkasse finanzierte Behandlung. Daher müssen bei der Gestaltung der Klassifikationssysteme neben der wissenschaftlichen Evidenz und der praktischen Umsetzbarkeit auch juristische und politische Dimensionen mitberücksichtigt werden, die in der Wissenschaft keine oder jedenfalls nur eine untergeordnete Rolle spielen sollten. Die kritische Analyse der Klassifikationssysteme, die wir hier vorstellen, ist aus einer rein wissenschaftlichen Sichtweise formuliert und erhebt nicht den Anspruch, den genannten komplexen Anforderungen an die Klassifikationssysteme vollständig gerecht zu werden.

Eine vollständige und systematische Klassifikation im Sinne einer Taxonomie psychischer Störungen ist an sich bereits ein Ziel der Wissenschaft. Darüber hinaus allerdings bildet eine solche Klassifikation die Grundlage von Forschung, in- 
dem sie die zu untersuchenden Phänomene zunächst beschreibt, in einer hochgradig operationalisierten Weise verbindlich definiert und zueinander in Beziehung stellt. Eine Untersuchung von Komorbidität (hier verstanden als das gleichzeitige oder zumindest zeitlich enge Zutreffen der Klassifikationskriterien mehrerer psychischer Störungen) etwa kann nur erfolgen, wenn die beiden Störungen hinreichend gut beschrieben und abgegrenzt sind. Daher bildet eine systematische Klassifikation psychischer Störungen die Grundlage für die wissenschaftliche Untersuchung und damit auch für das bessere Verständnis der Störungen sowie für die Entwicklung besserer Therapien. Dabei gilt natürlich auch immer, dass die Klassifikation von der Forschung beeinflusst wird, sodass Phänomenbeschreibungen immer wieder an den aktuellen Stand der Forschung angepasst werden müssen.

Auch über die Psychiatrie hinaus kommt der Klassifizierung psychischer Störungen eine entscheidende Rolle zu, nämlich in Bezug auf das Verständnis des menschlichen Geistes generell. Das Verständnis einzelner Funktionen eines komplexen Systems kann besonders gut anhand von ungewöhnlichen Reaktionen des Systems, um nicht zu sagen: an Fehlfunktionen, erfolgen. Das gilt bereits im Alltag: Über die (Existenz und) Funktionsweise einzelner Muskeln erfährt man oft erst dann etwas, wenn diese Muskeln etwa durch Überbelastung schmerzen oder nicht die gewohnte Arbeit verrichten. Auf ähnliche Weise funktioniert die Experimentelle Psychologie, die (grob vereinfacht) oft versucht, in einem experimentellen Umfeld ungewöhnliches Verhalten zu provozieren, anhand dessen sie dann auf die Funktionsweise des Geistes schließen kann. Genauso halten die ungewöhnlichen Phänomene, die wir im Bereich der psychischen Störungen antreffen, wertvolle Hinweise für das Verständnis des „normal“ funktionierenden menschlichen Geistes bereit. Dabei geht es nicht nur um das Verständnis einzelner Funktionen (z. B. der Wahrnehmung, für deren Verständnis Halluzinationen eine große Rolle spielen), sondern auch um das Zusammenwirken einzelner Funktionen bis hin zu einem generellen Bild der „Architektur des Geistes“.

Aus dieser Disziplinen-übergreifenden Perspektive ist die Frage, welche Erscheinungsformen psychischer Funktionen man als „krank“ ansehen sollte und welche nicht, nachgeordnet. Es geht also in der Wissenschaft, anders als in der Klassifikation von Erkrankungen, nicht in erster Linie um eine Klassifikation von Pathologien, sondern um eine Klassifikation von Phänomenen. Ob und inwiefern 
diese Phänomene dann als „pathologisch“ (im Sinne von „eine Krankheit anzeigend") anzusehen sind, ist eine andere Frage, die hier nicht im Vordergrund steht, die aber für die oben genannten Klassifikationssysteme von entscheidender Bedeutung ist. Um den menschlichen Geist anhand von ungewöhnlichen Phänomenen besser zu verstehen, ist es zunächst unerheblich, ob diese ungewöhnlichen Phänomene zu einem Leidensdruck oder einer Einschränkung der Funktionstüchtigkeit im Alltag führen - diese Frage kommt erst ins Spiel, wenn wir uns dafür interessieren, welche Kriterien „Leiden“ oder „Funktionstüchtigkeit im Alltag“ ausmachen. Wie entscheidend die letztgenannten Fragen werden können, zeigt die Tatsache, dass nach DSM-5 für das Vorliegen einer psychischen Störung in der Regel Einschränkungen der Alltags-Funktionen erforderlich sind, während dies in ICD-10 - und aller Wahrscheinlichkeit nach auch in ICD-11 - kein Kernkriterium für die Feststellung einer „Krankheit“ ist. Vor diesem Hintergrund soll hier die Debatte um den Begriff der „Pathologie“ oder der Grenzziehung zwischen „noch gesund“ und „schon krank“ ausgeklammert bleiben.

Das Ziel einer wissenschaftlichen Klassifikation psychischer Störungen ist also, eine möglichst gute Beschreibung von beobachtbaren Phänomenen psychischer Störungen zu liefern, die die Grundlage für das Verständnis dieser Phänomene selbst, aber auch für das Verständnis des menschlichen Geistes allgemein bilden können. Dafür ist eine systematische Klassifikation nötig, die die Phänomene einheitlich nach bestimmten Dimensionen einordnet. Sie sollte möglichst einfach sein, muss aber selbstverständlich komplex genug sein, um die relevanten Unterschiede darstellen zu können.

Sowohl in DSM-5 als auch in ICD-10 erfolgt die Klassifikation nach verschiedenen Dimensionen, die allerdings nicht hierarchisch-systematisch, sondern nebeneinander stehend eingesetzt werden. So sind etwa in ICD-10 verschiedene Störungen zu Gruppen zusammengefasst, und zwar u. a. nach folgenden Kriterien: Ätiologie (z. B. Störungen, die durch Missbrauch von Substanzen ausgelöst wurden), Zeitpunkt des Auftretens (z. B. Störungen, die im Kindesalter auftreten), betroffene „Funktionen“ (z. B. affektive Störungen, Intelligenzstörungen), Auftreten bestimmter Symptome (z. B. Schizophrenie). Offensichtlich schließen sich diese Kriterien nicht wechselseitig aus, sodass eine Zuordnung der Gruppen nicht ohne 
weiteres erfolgen kann - hier erfolgt dann eine definitorische Setzung, indem beispielsweise Hierarchisierungs- oder Ausschlussregeln eingeführt werden. ${ }^{1}$

Wenn wir allerdings davon ausgehen, dass die Störung einer bestimmten geistigen Funktion grundsätzlich immer dieselben Effekte hat, dann spielen solche Kriterien wie Zeitpunkt des Auftretens oder die Ätiologie der Funktionsstörung zunächst keine Rolle. Vielmehr ist zunächst eine systematische Klassifikation der beobachtbaren Effekte der Störungswirkung (Symptome) anhand von standardisierten Kriterien notwendig, die einen Rückschluss auf zugrundeliegende Funktionsstörungen erst ermöglicht. Natürlich ist die Realität viel komplexer, da nicht nur ein bestimmter „klinischer“ Effekt durch verschiedene Funktionsstörungen hervorgerufen werden kann, sondern auch, weil sich in der Interaktion von verschiedenen Faktoren dieselbe Funktionsstörung durchaus unterschiedlich äußern kann oder verschiedene Störungseinwirkungen zu identischen „klinischen“ Erscheinungsweisen der Funktionsstörung führen können. Umso wichtiger ist es, eine Beschreibung und Klassifikation der Symptome zu finden, die solche Interaktionen auffindbar macht und nicht durch die unsystematische Anwendung verschiedener Kriterien verschleiert.

Tatsächlich entwickeln sich die Klassifikationen der APA (DSM) und der WHO (ICD) in Richtung eines solchen „dimensionalen“ Ansatzes, der nicht von unabhängig von den Ursachen bestehenden Krankheitsbildern ausgeht (also anosologisch ist), sondern Symptom-orientiert vorgeht (Zielasek/Gaebel 2008; Gaebel/ Zielasek 2009). Diese Entwicklung ist allerdings bei langem noch nicht abgeschlossen. Dieser Beitrag hat zum Ziel, nachzuweisen, dass Frames ein gutes Mittel darstellen, um diese Entwicklung weiter zu unterstützen, indem Sie eine übersichtliche Darstellung verschiedener Symptome erlauben und gleichzeitig die systematischen Zusammenhänge zwischen verschiedenen Funktionen des Geistes deutlich werden lassen.

1 Eine solche Ausschlussregel könnte z. B. lauten: Eine Schizophrenie wird nicht diagnostiziert, wenn die Symptome auf die Wirkung von Rauschmitteln zurückzuführen sind. 


\section{Vorteile der Frame-Darstellung}

Frames sind rekursive Attribut-Wert-Strukturen, die die Struktur von Repräsentationen abbilden (Barsalou 1992; Petersen 2007). Der zu beschreibende Begriff, in unserem Fall z. B. das Symptom, kann als zentraler Knoten dargestellt werden, der durch bestimmte Attribute, durch Pfeile dargestellt, charakterisiert wird. Attribute sind Funktionen, die dem Begriff jeweils einen bestimmten Wert zuordnen. Der Wert kann wiederum durch weitere Attribute spezifiziert werden. Abb. 1 zeigt als Beispiel einen stark vereinfachten Frame von intrusiven Gedanken, die bei Patienten mit Zwangsstörungen auftreten und zu den typischen Zwangshandlungen führen.

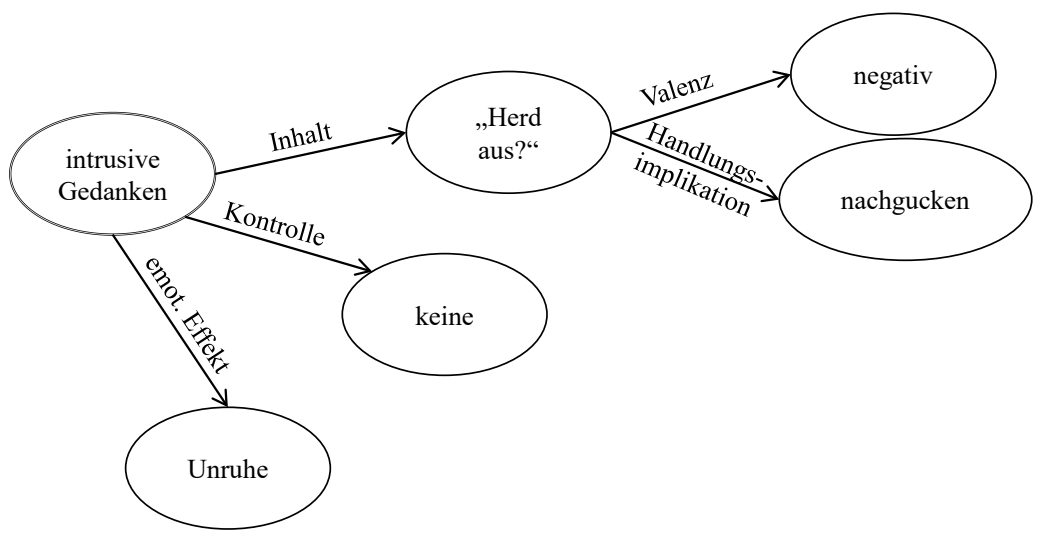

Abb. 1: Vereinfachte Frame-Darstellung von intrusiven Gedanken

In diesem Frame wird übersichtlich dargestellt, dass sich intrusive Gedanken dadurch auszeichnen, dass sie Unruhe auslösen, dass die Patienten das Auftreten dieser Gedanken nicht kontrollieren können und dass solche Gedanken einen typischen Inhalt haben. In unserem Beispiel handelt es sich um den Gedanken, dass der Herd nicht ausgeschaltet wurde. Die Angabe im Inhalts-Knoten zeigt an, zu welchem Inhaltstyp ein bestimmter intrusiver Gedanke gehört. Natürlich gibt es auch andere intrusive Gedanken mit anderen Inhalten. Wenn ausgedrückt werden soll, dass zwar jeder intrusive Gedanke einen bestimmten Inhalt hat, dieser Inhalt aber zu verschiedenen Typen gehören kann, wird in der Frame-Darstellung 
der Inhalts-Knoten schlicht leer gelassen. Weiterhin wird dargestellt, dass die Valenz des Inhalts des Gedankens negativ ist und dass der Inhalt bestimmte Implikationen für das Verhalten hat, in diesem Fall die Implikation, nachzugucken, ob der Herd wirklich noch eingeschaltet ist. Während die spezifische Handlungsimplikation mit dem Inhaltstyp variiert (und demnach dieser Knoten bei fehlender Typ-Einordnung des Inhalts-Knotens leer bleiben würde), können wir davon ausgehen, dass alle intrusiven Gedanken, die mit einer Zwangsstörung verbunden sind, eine negative Valenz haben. Dieser Wert bleibt also im Frame erhalten, sodass sich aus dieser Spezifikation dieses Wertes bereits eine Einschränkung für mögliche Typen im Inhalts-Knoten ergibt.

Offensichtlich haben wir uns in diesem Beispiel auf eine psychologische Beschreibungsebene beschränkt. Ebenso gut lassen sich allerdings andere Dimensionen in den Frame einfügen, etwa eine neuronale, eine genetische und auch eine soziale Dimension. So könnte etwa der als Unruhe bezeichnete emotionale Effekt weiter spezifiziert werden durch physiologische Attribute wie Blutdruck, Puls und Schweißproduktion, während die negative Valenz des Inhaltes in Beziehung gesetzt werden könnte zu sozialen Normen.

Es wird schnell ersichtlich, dass ein vollständiger Frame in diesem Sinne äußerst komplex werden kann. Durch die Beschränkung auf funktionale Attribute, die eine eindeutige Zuordnung von Werten erfordern, wird der Frame allerdings nicht zu einer beliebigen grafischen Darstellung, sondern erfasst gezielt die spezifischen Zusammenhänge zwischen einzelnen Faktoren. Zusätzlich können bestimmte Attribute bestimmten „Bereichen“ oder funktionalen Einheiten zugeordnet werden, wie z. B. dem Denken oder der Handlungssteuerung. Dadurch werden nicht nur systematische Zusammenhänge zwischen einzelnen Funktionen deutlich, sondern auch Auswirkungen von einzelnen Funktionsstörungen auf unterschiedliche Bereiche sowie generell Zusammenhänge zwischen verschiedenen Dimensionen. Auf diese Art und Weise kann die komplexe Einbettung von einzelnen Funktionen in einen größeren Zusammenhang auf verschiedenen Beschreibungsebenen systematisch dargestellt werden. Dies führt nicht nur zu neuen Erkenntnissen und Hypothesen über mögliche Entstehungsmechanismen, sondern es erlaubt auch eine systematische Vergleichbarkeit von Symptomen. 
Im Folgenden soll nun am Beispiel von Fremdbeeinflussung und Wahn schrittweise gezeigt werden, wie eine Frame-Darstellung aufgebaut werden kann und welche Perspektiven der Klassifizierung sich daraus ergeben.

\section{Beispiel Fremdbeeinflussung}

Bei dem Phänomen der „Fremdbeeinflussung“ berichten Betroffene, dass einige ihrer Handlungen oder Gedanken nicht von ihnen gemacht werden, sondern von anderen Menschen oder fremden Mächten. Wir beziehen uns im Folgenden auf Fälle, in denen Bewegungen von den Betroffenen bisweilen als fremdgesteuert beschrieben werden.

Im deutschen Sprachraum wird dieses Symptom als „Ich-Störung“ klassifiziert, zusammen mit anderen Symptomen wie Gedankeneingebung, Derealisation, etc. Der zugrundeliegende Gedanke ist, dass die Grenzen des erlebten „Ich“, zu dem u. a. die eigenen Handlungen und die eigenen Gedanken gehören, verschwimmen, und eine normale Trennung von "Ich“ und Umwelt nicht mehr stattfindet. ${ }^{2}$ In DSM-5 wird diese Symptomgruppe unter den Wahn-Phänomenen klassifiziert (englischer Name: delusion of control; DSM-5, S. 87). Wahn wird dabei aufgefasst als falsche Überzeugung, die auf fehlerhaften Schlüssen die umgebende Welt betreffend basiert und an der trotz überwältigender Gegenevidenz festgehalten wird. ${ }^{3}$ Die angelsächsische Gruppierung von Symptomen legt also den Fokus auf den Überzeugungs-Charakter und auf fehlerhafte „Denkmechanismen“. Während auf der einen Seite die Betonung des Inhalts dazu führen wird, die Ursache für die Äußerungen der Betroffenen v. a. in abnormen „Ich“-Erfahrungen zu suchen, führt eine Fokussierung auf die Besonderheiten der Überzeugung dazu, die Ursache in einer Störung des „Überzeugungssystems“ zu suchen. Darüber hinaus hat sich in den letzten Jahrzehnten ein neurokognitives Modell der Fremdbeeinflussung etabliert, das unter dem Namen „Komparator-Modell“ bekannt geworden ist (Frith 1992; Synofzik/Vosgerau/Newen 2008a). Dieses Modell ist zunächst ein allgemeines Modell der Motorik-Steuerung, das grob skizziert Folgendes behauptet (vgl. auch Abb. 2): Vor jeder (absichtlichen) Bewegung steht eine Intention bzw.

2 Eine kritische Auseinandersetzung mit dem Begriff des „Ich“ findet sich in Beckermann (2010).

3 „Delusions are fixed beliefs that are not amenable to change in light of conflicting evidence." (American Psychiatric Association 2013, 87). 
ein Ziel, die bzw. das nicht unbedingt bewusst vorliegen muss. Aufgrund dieser Intention werden ein Bewegungsplan und schließlich ein Motorik-Kommando erstellt, das an die Muskeln gesendet wird, sodass die Bewegung ausgeführt wird. Dabei wird von dem Motorik-Kommando eine Kopie erstellt (die sog. „Efferenzkopie"), ${ }^{4}$ aus der eine Vorhersage errechnet wird über die auszuführende Bewegung. Diese Vorhersage kann nun ständig von einem „Komparator“ mit der durch verschiedene Sinne registrierten tatsächlichen Bewegung verglichen werden. Sollte es zu Abweichungen zwischen Vorhersage und tatsächlicher Bewegung kommen, kann nicht nur die Bewegung bereits während der Ausführung korrigiert werden, sondern die Mechanismen zur Erstellung des Motorik-Kommandos können auch auf längere Sicht verbessert werden.

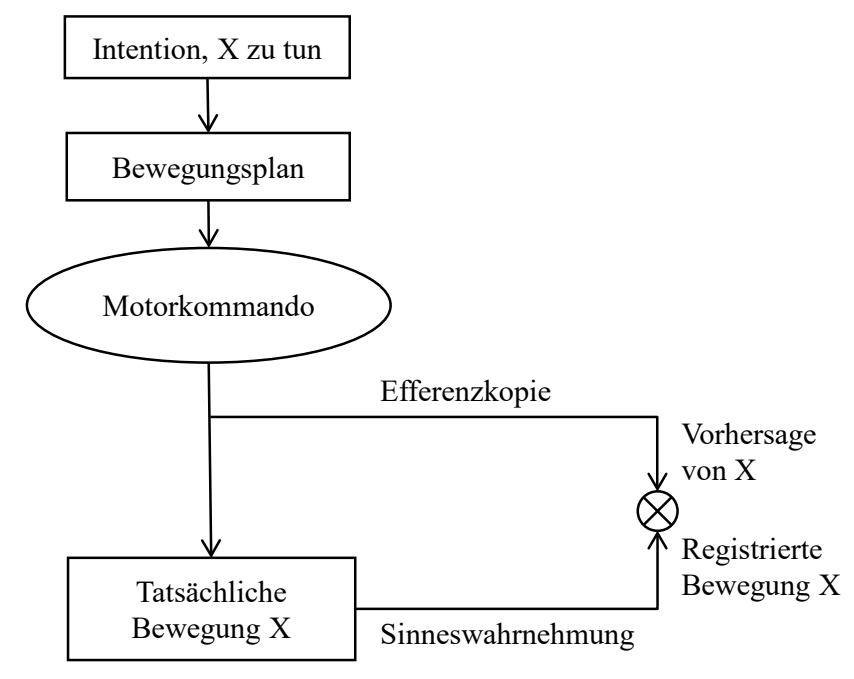

Abb. 2: Das Komparator-Modell (vereinfacht); bei dieser Darstellung handelt es sich um einen Flow-Chart, der die Informationsflüsse abbilden soll, und nicht um einen Frame mit funktionalen Attributen.

Das Komparator-Modell stellt nach Frith (Frith/Blakemore/Wolpert 2000; Frith 2005) eine einfache Erklärung für unser alltägliches Erleben der Urheberschaft

4 Die Idee der Efferenzkopie stammt bereits aus den 1950er Jahren (von Holst/Mittelstaedt 1950; Sperry 1950). 
unserer Bewegungen bereit: Wenn der Komparator eine Übereinstimmung zwischen Vorhersage und tatsächlicher Bewegung registriert, dann schreiben wir uns die Initiierung und Kontrolle, also die Urheberschaft für diese Bewegung selbst zu. Wenn allerdings eine Abweichung registriert wird, dann können wir davon ausgehen, dass (z. B. aufgrund von äußeren Einflüssen) die Bewegung nicht bzw. nicht vollständig von uns initiiert und kontrolliert wurde, sodass in diesem Fall kein Gefühl der Urheberschaft entsteht, sondern diese fremdzugeschrieben wird (das ist z. B. der Fall, wenn jemand anders meinen Arm bewegt). Im Fall der Fremdbeeinflussung, so diese Theorie, liegt eine noch näher zu spezifizierende Störung in diesem Mechanismus vor (etwa in der Vorhersage, in der Wahrnehmung der Bewegung oder im Komparator selbst), sodass fälschlicherweise eine Abweichung zwischen Vorhersage und registrierter Bewegung gemeldet wird, die zu einer falschen Fremdzuschreibung der Urheberschaft führt.

Mit dem Komparator-Modell haben wir nun eine dritte Möglichkeit der Klassifizierung des Symptoms, nämlich als Störung der Motorik-Steuerung. Offensichtlich schließen sich die verschiedenen Erklärungen aber nicht vollständig aus: Eine Störung in der Motorik-Steuerung könnte tatsächlich zu einer ungewöhnlichen Urheberschafts-Erfahrung führen, und da das Erleben der Urheberschaft von Bewegungen einen wichtigen Teil unseres „Ich“ ausmacht, zu veränderten „Ich“-Erfahrungen. Gleichzeitig könnte eine solche Erfahrung zu Überzeugungen führen, die aufgrund einer Störung der Denkmechanismen trotz Gegenevidenz aufrechterhalten wird. ${ }^{5}$ Ein solches Modell wird zwei-Stufen-Modell genannt, da auf der ersten Stufe eine ungewöhnliche Erfahrung steht, die auf der zweiten Stufe in das Überzeugungssystem eingefügt wird.

Tatsächlich gibt es einige Kritikpunkte am Komparator-Modell als alleinige Erklärung für Fremdbeeinflussung (Synofzik/Vosgerau/Newen 2008a), von denen hier nur wenige exemplarisch genannt werden sollen: Ein Komparator kann nur zwischen „ich“ und „nicht-ich“ unterscheiden, aber er kann nicht zu dem Gefühl führen, dass ausgerechnet mein Nachbar meine Bewegungen steuert. Zudem sollte eine generelle Störung der Bewegungssteuerung zu einer generellen Unfähigkeit, sich Urheberschaft zuzuschreiben, führen - tatsächlich aber tritt die

5 Die ursprüngliche Formulierung des Komparator-Modells schließt ein solches zwei-Stufen-Modell zwar aus, aber die Beschränkung des Komparator-Modells auf die erste Stufe ist offensichtlich einfach zu bewerkstelligen. 
fälschliche Fremdzuschreibung nur für manche Bewegungen auf. Aber auch im Detail treten Probleme mit dem Komparator-Modell auf, das die Komplexität der verschiedenen Sinneswahrnehmungen (visuell, kinästhetisch,...) und die kontextabhängige Relevanz der einzelnen Faktoren nur unzureichend berücksichtigen kann.

Gleichzeitig kann aber gezeigt werden, dass von einer Schizophrenie Betroffene tatsächlich Auffälligkeiten in der Bewegungs-Steuerung zeigen (Synofzik u. a. 2010; Synofzik/Voss 2010). Darüber hinaus werden auch bei vielen anderen psychischen Störungen Veränderungen der Motorik beobachtet, die anzeigen, dass hier diagnoseübergreifend ein allgemeines Prinzip wirkt. Dies hat beispielsweise dazu geführt, dass in DSM-5 Veränderungen der (Psycho-)Motorik mittlerweile einen eigenständigen diagnostischen Stellenwert in der Reihe der dimensionalen Symptomklassifikations-Merkmale bekommen haben. Dies weist darauf hin, dass die Grundidee des Komparator-Modells nicht falsch ist, auch wenn das Modell modifiziert und erweitert werden muss. Weiterhin ist auch bekannt, dass beispielsweise bei Patienten mit einer Schizophrenie eine Reihe von Auffälligkeiten im Bereich der Urteilsbildung auftritt (McKay 2012; Moritz/Woodward 2005; Woodward u. a. 2006), sodass auch dieser Faktor nicht unberücksichtigt bleiben darf. Ein zwei-Stufen-Modell der Urheberschaft liegt also nahe (Davies u. a. 2001).

Das „multifaktorielle Gewichtungsmodell“ der Urheberschaft (Synofzik/Vosgerau/Newen 2008b) geht daher von zwei Ebenen aus (siehe auch Abb. 3): Auf der Ebene der „Urheberschafts-Gefühle“ liegen perzeptuelle Repräsentationen vor, die ein Eindruck der eigenen Urheberschaft vermitteln. Dieses „Gefühl“ basiert auf verschiedenen Faktoren („cues“), die Urheberschaft anzeigen können, wie etwa „feed-forward Signale“ (das ist im Wesentlichen die Vorhersage im Komparator-Modell), Informationen aus der Propriozeption (z. B. Kinästhetik) und sensorisches Feedback (z. B. visuelle Information). Je nach Situation können diese verschiedenen Faktoren Informationen unterschiedlicher Qualität (v. a. in Hinblick auf Genauigkeit und Reliabilität) liefern, sodass sie gemäß der Situation jeweils gewichtet werden. Wenn ich mich z. B. in einem dunklen Raum bewege, kann und sollte ich mich nicht auf die visuellen Informationen zu meiner Bewegung verlassen, die hier nur sehr spärliche und wenig verlässliche Informationen liefern; in diesem Fall würde das visuelle Feedback ein sehr kleines Gewicht bekommen, während z. B. die Propriozeption stärker gewichtet würde. 
Auf der Ebene des Urheberschafts-Urteils findet wiederum eine gewichtete Integration verschiedener Faktoren statt, wobei das Gefühl der Urheberschaft hierbei nur einen von vielen Faktoren darstellt. Dazu kommen Faktoren wie Hintergrundüberzeugungen, Kontext-Informationen, soziale Normen etc. All diese Faktoren spielen eine Rolle dabei, die Urheberschaftszuschreibung zu generieren, die wir dann als Urteil auch äußern. Dabei mag es sein, dass in den meisten Alltagsfällen der Inhalt des Gefühls mit dem Inhalt des Urteils übereinstimmt: Meistens haben wir nicht den Eindruck, dass unser Gefühl uns täuscht. Allerdings kann es in bestimmten Situationen Abweichungen des Urteils vom Gefühl geben. Stellen Sie sich z. B. vor, Sie sind allein in einem Raum mit einem Tisch, auf dem eine Vase steht. Plötzlich fällt die Vase herunter. Obwohl Sie nicht das Gefühl haben, etwas gemacht zu haben, werden Sie sich wahrscheinlich diesen vermeintlichen Effekt Ihrer Bewegung selbst zuschreiben, aus dem einfachen Grund, dass Sie wissen, dass Vasen nicht von alleine von Tischen fallen und dass niemand anders im Raum ist. In einem solchen Fall kann es also dazu kommen, dass wir zu der Überzeugung kommen, dass wir die Urheber eines Effekts sind, obwohl wir nicht das Gefühl haben, etwas getan zu haben. Der Grund ist, dass Hintergrundüberzeugungen und Kontextwissen den Inhalt des Gefühls „überschreiben“ können, ohne dabei das Gefühl selbst zu ändern.

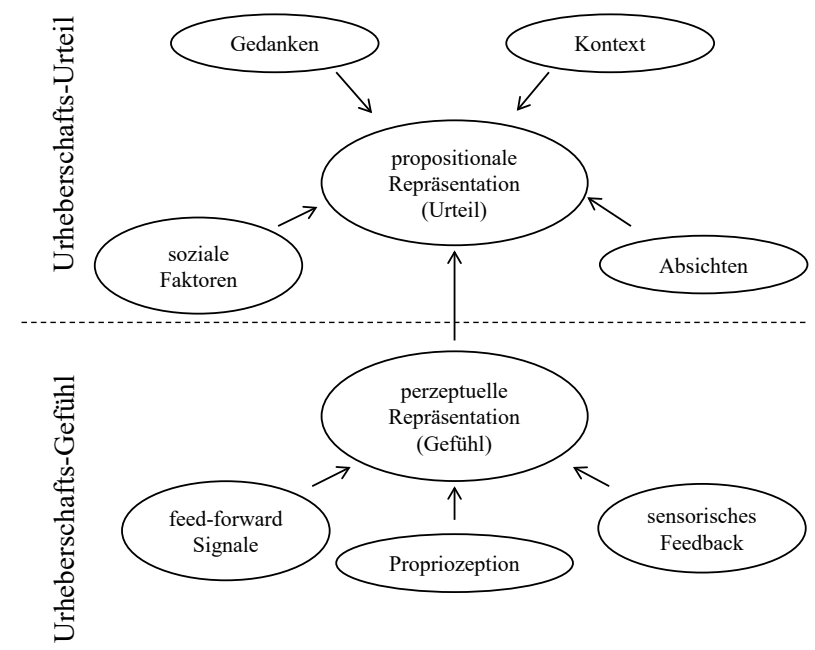

Abb. 3: Das multifaktorielle Gewichtungsmodell; auch bei dieser Darstellung handelt es sich nicht um einen Frame, sondern um ein Flow-Chart. 
Das multifaktorielle Gewichtungsmodell der Urheberschaft erlaubt damit eine etwas komplexere Erklärung der Fremdbeeinflussung: Eine Störung kann auf ganz verschiedenen Ebenen vorliegen und trotzdem zu ähnlichen klinisch beobachtbaren Effekten führen. Zudem lassen sich bei Personen mit derartigen Fremdbeeinflussungs-Symptomen systematische Verzerrungen der feed-forward-Signale und eventuell der propriozeptiven Wahrnehmung nachweisen (siehe z. B. Lindner u. a. 2005; Shergill u. a. 2005; Synofzik u. a. 2010). Vor diesem Hintergrund scheint die folgende Hypothese gerechtfertigt: Ein Pathomechanismus der Fremdbeeinflussungs-Symptome könnte dann eine mangelnde Beachtung der „internen“ Signale und eine Übergewichtung von „externen“ Signalen sein, die von der Tatsache herrührt, dass die internen Signale nur verzerrte und daher unzuverlässige Informationen liefern. Das könnte wiederum dazu führen, dass sich Betroffene hauptsächlich auf „externe“ Faktoren bzw. auf ihre Hintergrundüberzeugungen verlassen, um Urheberschafts-Urteile zu bilden. Das kann in manchen Situationen zur falschen Fremdzuschreibung von Urheberschaft führen (mit dem daraus folgenden klinischen Phänomen der „Fremdbeeinflussung“), es kann aber auch in experimentellen Situationen zu Selbstzuschreibungen für Bewegungen führen, für die gesunde Probanden sich keine Urheberschaft zuschreiben.

Um diese komplexe Lage übersichtlicher darzustellen, soll hierzu nun eine Frame-Darstellung vorgestellt werden. Wie oben bereits erwähnt, bestehen Frames nicht nur aus Kreisen, die mit Pfeilen verbunden sind. Vielmehr stellen die Pfeile Attribute dar, die als Funktionen aufgefasst werden. Daher können die Darstellungen aus Abb. 2 und 3 nicht ohne weiteres übernommen werden, da es sich bei diesen Abbildungen nicht um Frames, sondern vielmehr um Flow-Charts handelt, die den Informationsfluss anzeigen, aber keine Attribute. Diese formale Einschränkung von Frames wird uns dazu zwingen, die einzelnen Faktoren noch genauer zu bestimmen.

Zunächst soll die Grundidee des Komparator-Modells der Motorik-Steuerung dargestellt werden (Abb. 4): In dieser Darstellung können die einzelnen Komponenten des Komparator-Modells dargestellt werden, wobei ihr jeweiliger funktionaler Zusammenhang deutlich wird. Im Gegensatz zu der vereinfachten Darstellung in Abb. 2 haben wir nun zwei Komparatoren unterschieden: Ein Komparator gleicht vorhergesagten Zustand mit wahrgenommenem Zustand ab, so wie oben erläutert. Ein weiterer Komparator überprüft, ob das eigentlich intendierte Ziel 
überhaupt erreicht wurde. Diesen Komparator hatten wir in der obigen vereinfachten Version nicht erwähnt; auch dieser Komparator wird zur Erklärung von Fremdbeeinflussung herangezogen (vgl. etwa Carruthers 2012). Letztendlich führt aber die Einführung von mehreren Komparatoren zu dem zusätzlichen Problem, dass dann entschieden werden muss, welcher Komparator unter welchen Umständen der relevante ist (Vosgerau/Synofzik 2012). Da dieses Problem allerdings vom multifaktoriellen Gewichtungsmodell behoben wird, und da es hier nicht so sehr um die Details der Erklärung von Fremdbeeinflussung, sondern mehr um die Vorteile einer Frame-Darstellung geht, möchten wir nicht weiter auf solche Einzelheiten eingehen.

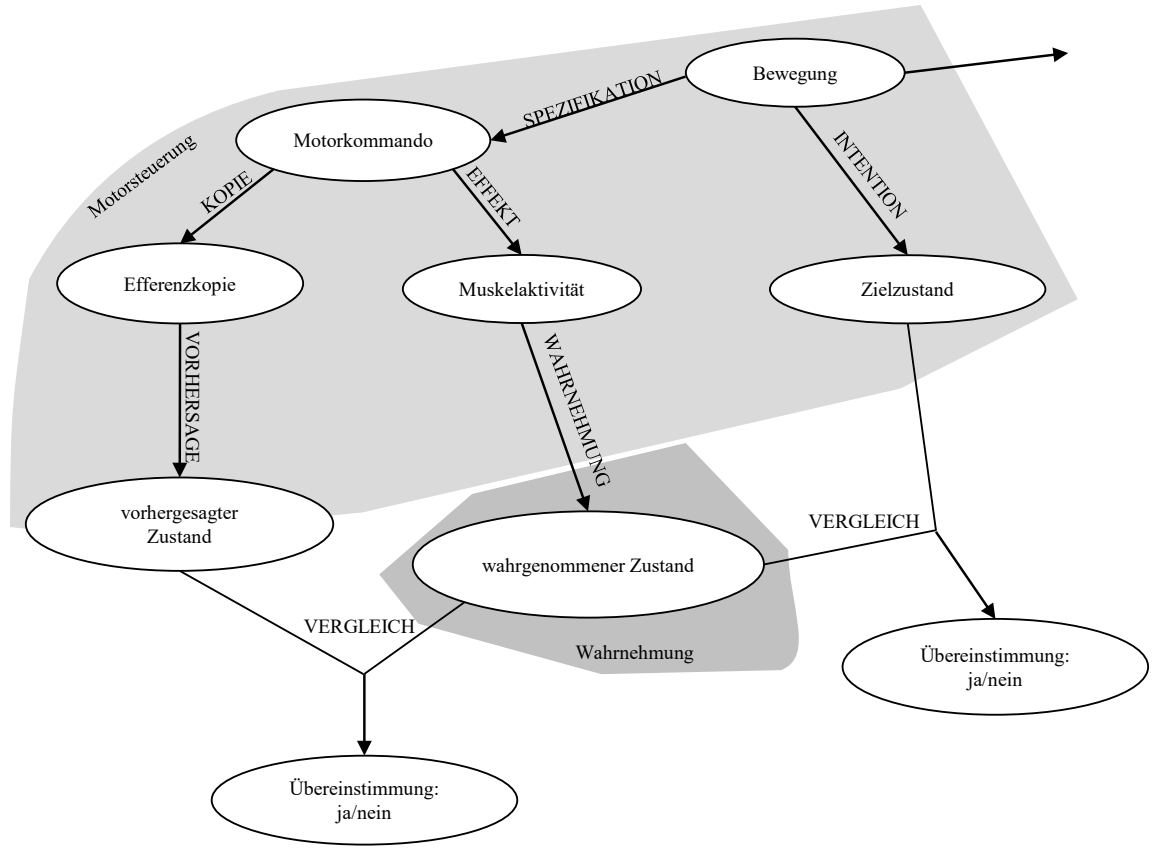

Abb. 4: Die Grundidee des Komparator-Modells als Frame; bei diesem Frame werden auch Attribute eingeführt, die zwei Werte eindeutig auf einen Wert abbilden, die also eine Funktion mit zwei Argumenten darstellen.

Zusätzlich haben wir in Abb. 4 die verschiedenen Bereiche bzw. funktionalen Einheiten des Geistes durch graue Hintergrundflächen markiert. Diese Zuordnung 
ließe sich zwar auch relativ einfach durch Attribute bewerkstelligen, würde aber zu einer Unübersichtlichkeit führen. Interessant an dieser Zuordnung ist nun, dass wir nicht nur eine spezifische Störung einem bestimmten Bereich zuordnen können, sondern gerade, dass die genauen funktionalen Zusammenhänge zwischen verschiedenen Bereichen sichtbar werden und dadurch die Auswirkungen einer zunächst eng umgrenzten Störung auf ganz andere Bereich nachvollziehbar werden.

Wir können nun (zumindest in erster Näherung) die Vergleichsergebnisse der beiden Komparatoren (deren Existenz vom multifaktoriellen Gewichtungsmodell auch nicht bestritten wird) als zwei der Faktoren („cues“) ansehen, die unser Gefühl von Urheberschaft mitbestimmen. So lässt sich der eben beschriebene Frame erweitern im Sinne des multifaktoriellen Gewichtungsmodells (siehe Abb. 5).

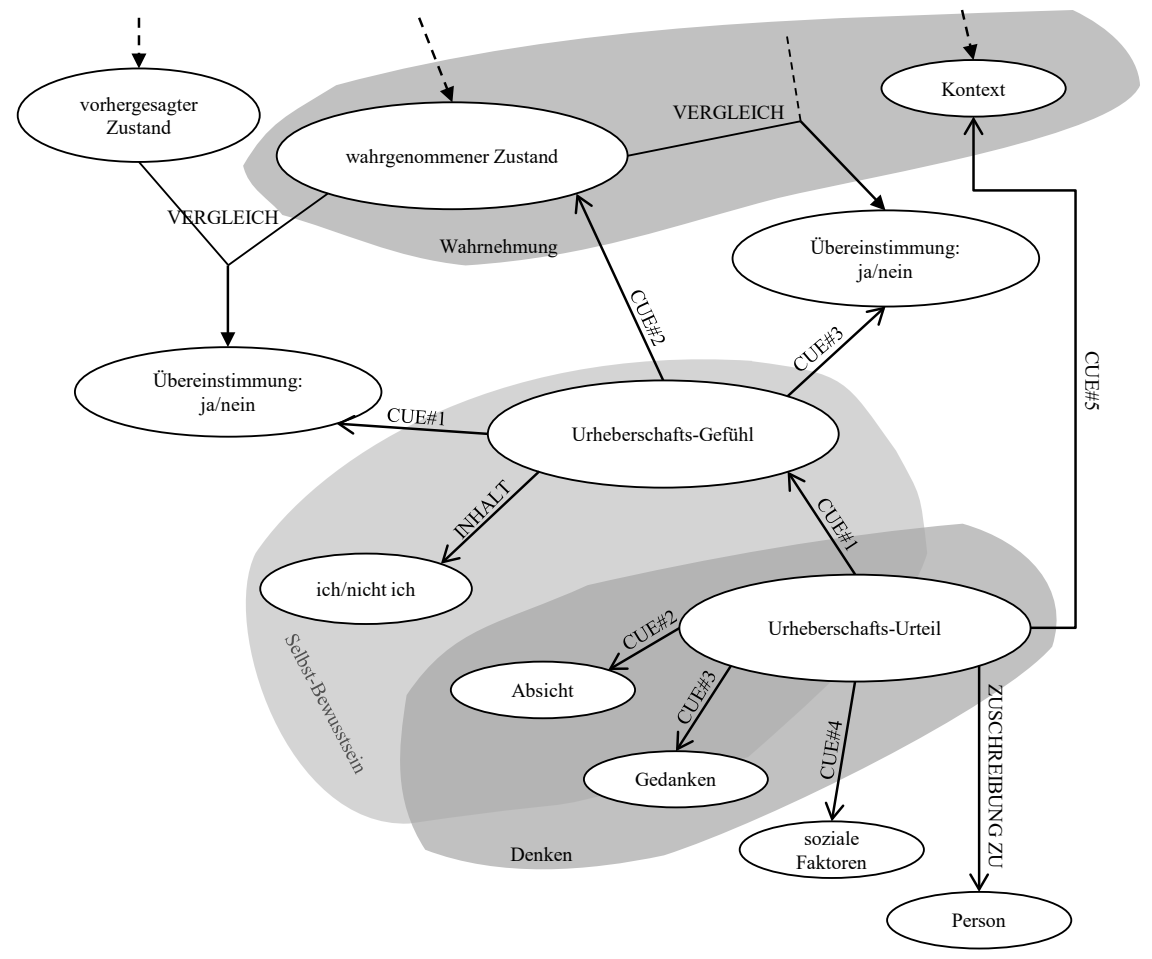

Abb. 5: Frame-Darstellung multifaktorielles Gewichtungsmodell (Fortsetzung zu Abb. 4) 
Nach diesem Frame basiert das Urheberschafts-Gefühl auf drei Faktoren: dem wahrgenommenen Zustand und den beiden Komparator-Ergebnissen. Es ist selbst einer von fünf Faktoren, die das Urheberschafts-Urteil bestimmen. Während der Inhalt des Gefühls nur eine Unterscheidung zwischen „ich“ und „nichtich“ zulässt, kann auf der Ebene des Urteils die Urheberschaft einer bestimmten Person (inkl. sich selbst) zugeschrieben werden. Dabei können die verschiedenen Knoten (teilweise) verschiedenen Bereichen zugeordnet werden. Das komplexe Zusammenspiel von Motorik, Wahrnehmung, nicht-begrifflichem und begrifflichem Selbst-Bewusstsein ${ }^{6}$ sowie dem Denken allgemein werden so sichtbar. So wird verständlich, dass sich die oben zitierten unterschiedlichen Einordnungen der Fremdbeeinflussung nicht ausschließen, sondern lediglich unterschiedliche Aspekte des Symptoms (über-)betonen: Tatsächlich ist es plausibel, dass eine Störung im motorischen System bzw. der Wahrnehmung der eigenen Bewegungen vorliegt, die zu einem abnormalen Gefühl führt, das zum Selbst-Bewusstsein gerechnet werden kann (ein Aspekt eines „Ich“-Gefühls). Darüber hinaus führt dieses Gefühl dann aber zu einem Urteil, das klarerweise dem Bereich des Denkens (und bei Selbstzuschreibung auch dem Selbst-Bewusstsein) zugeordnet werden kann.

Ein Vorteil der Frame-Darstellung ist die rekursive Struktur von Frames. Das bedeutet, dass jeder Knoten weiter spezifiziert werden kann durch weitere Attribute. So könnte man etwa den wahrgenommenen Zustand weiter spezifizieren, indem man unterschiedliche Sinnesmodalitäten unterscheidet. Plausiblerweise spielen beim Entstehen des Urheberschafts-Gefühls vor allem propriozeptive (kinästhetische) und visuelle Wahrnehmungen eine Rolle, aber auch andere Sinnesmodalitäten wie Tastsinn und Gehör müssen zumindest in bestimmten Situationen miteinbezogen werden. In Abb. 5 haben wir bisher auch die unterschiedliche Gewichtung der einzelnen „cues“ vernachlässigt. Die Grundidee ist, dass je nach Kontext die einzelnen „cues“ unterschiedliche Reliabilität besitzen (bei Dunkelheit spielt visuelle Wahrnehmung z. B. kaum eine Rolle). Die Gewichtung ist daher eine Funktion von Kontext und „cue“. Eine Darstellung dieser Idee findet sich in Abb. 6, wobei wir aus Gründen der Übersichtlichkeit darauf verzichten,

6 Vgl. zu dieser Unterscheidung Vosgerau (2009a; 2009b; 2009c). 
einen kompletten Frame mit allen Aspekten abzubilden. Aufgrund der Rekursivität ist aber leicht ersichtlich, wie die dargestellten Teilframes jeweils zusammenzusetzen sind.

Auch bei der Gewichtung der einzelnen „cues“ müsste man für eine umfassende Darstellung noch weiter ins Detail gehen. So ist es plausibel anzunehmen, dass die Gewichtung nach einem Bayesianischen Modell funktioniert, in das neben dem Kontext auch frühere Erfahrungen und Vorannahmen einfließen (Synofzik/Vosgerau/Lindner 2009). Da das Prinzip der Rekursivität und der dadurch entstehenden Möglichkeit der inkrementellen Verfeinerung der Darstellung bereits durch ein Beispiel gezeigt wurde, verzichten wir auf eine nähere Diskussion dieses Punktes.

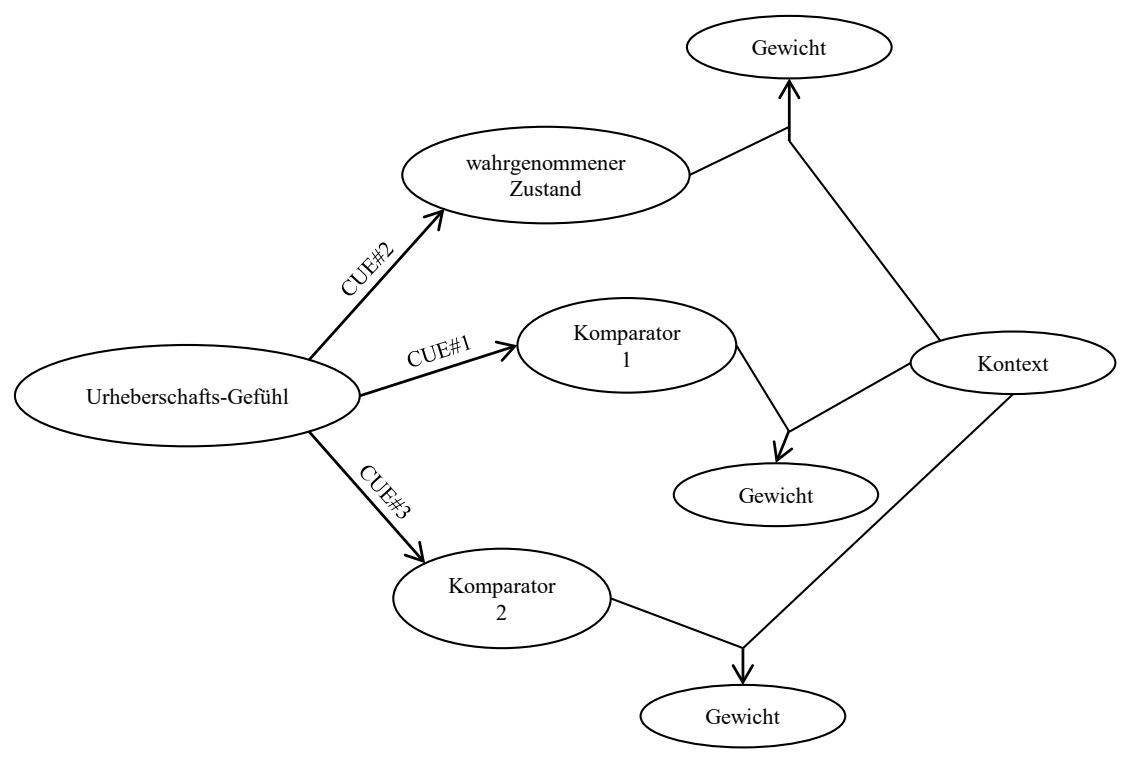

Abb. 6: Gewichtung von einzelnen „cues“ in Abhängigkeit des Kontextes

Ein weiterer interessanter Punkt, der in den bisherigen Frames nur unzureichend dargestellt ist, betrifft die Urteilsbildung selbst. Nach der gängigen angelsächsischen Einteilung handelt es sich beim Fremdbeeinflussungs-Symptom um einen Wahn, welcher wiederum als Urteil mit bestimmten Eigenschaften definiert ist 
(siehe oben). Eine weitere Präzisierung dieses Punktes erlaubt es uns daher, das Symptom der Fremdbeeinflussung in einen größeren Rahmen einzuordnen. Wir wenden uns daher nun einer kurzen Diskussion von Wahn im Allgemeinen zu.

\section{Beispiel Wahn}

Als Wahn werden gewöhnlich (z. B. nach DSM-5) Überzeugungen bezeichnet, die falsch sind, die auf fehlerhaften Schlüssen die umgebende Welt betreffend basieren und an denen trotz überwältigender Gegenevidenz festgehalten wird. Weiterhin werden meist Überzeugungen vom Wahn ausgeschlossen, die die Mehrheit der sozialen Gruppe der betreffenden Person hat (American Psychiatric Association 2013, 819). Letztere Einschränkung hat vor allem zum Ziel, kulturell oder religiös motivierte Überzeugungen nicht zu pathologisieren. Da es, wie oben bereits ausgeführt, bei einer wissenschaftlichen Klassifikation aber nicht in erster Linie darum geht, pathologische Fälle von nicht-pathologischen Fällen zu unterscheiden, werden wir auf dieses Kriterium keine Rücksicht nehmen. Darüber hinaus sind einige Punkte der Standard-Definition kritikwürdig. So ist z. B. fraglich, ob der Inhalt einer Wahnüberzeugung immer falsch sein muss, um als Wahn zu gelten. Es gibt Berichte von Personen, die unter wahnhafter Eifersucht litten, insofern sie keinerlei Anhaltspunkt oder Grund zur Eifersucht hatten. Trotzdem stellte sich ihre Überzeugung, dass sie von ihrem Partner betrogen werden, als zufällig wahr heraus (Enoch 1991). Die Tatsache, dass die wahnhafte Überzeugung kontingenterweise wahr ist, ändert also nichts an dem Status der Überzeugung als Wahn, wenn die entsprechende Überzeugung jeder Rechtfertigung entbehrt. Auch andere Punkte der Standard-Definition werden kontrovers diskutiert (vgl. Soom/Vosgerau 2015).

Wir wollen nun kurz einen eigenen Vorschlag zur Charakterisierung von (monothematischem) Wahn skizzieren (Soom/Vosgerau 2015), der die Frage nach der Pathologie völlig außer Betracht lässt. Wir vertreten dabei ein zwei-Stufen-Modell (vgl. Davies u. a. 2001), dass davon ausgeht, dass jedem monothematischen (also auf ein bestimmtes „Thema“ begrenzten) Wahn eine ungewöhnliche Erfahrung zugrunde liegt. Diese Erfahrung ist für die Betroffenen verstörend und verlangt nach einer Erklärung. Betroffene stellen zunächst eine Hypothese zur Er- 
klärung dieser Erfahrung auf, die sie immer wieder bestätigt finden. Der Denkprozess, der zur (dann wahnhaften) Überzeugung führt, ist also ein zunächst normaler abduktiver Denkprozess, ${ }^{7}$ der an sich nicht pathologisch ist. Allerdings muss der Gedanke derart sein, dass er tatsächlich das Auftreten der ungewöhnlichen Erlebnisse erklärt. Ein einfaches klinisches Beispiel ist das Auftreten von akustischen Halluzinationen in Form von imperativen Stimmen, die sich Betroffene im Rahmen einer wahnhaften Fehlinterpretation so erklären, dass $\mathrm{Au}$ ßerirdische, von denen man verfolgt wird, die Urheber dieser Stimmen seien. Jedoch kann der Wahn auch ohne solche Fehlwahrnehmungen auftreten: Beispielsweise sieht ein von einem Verfolgungswahn Betroffener im Fernsehen, wie sich der Nachrichtensprecher die Krawatte zurechtzieht. Der Betroffene wird verunsichert, da dies ein ungewöhnlicher Vorgang ist. Im Rahmen eines Verfolgungswahns interpretiert der Betroffene fälschlicherweise diese - richtige - Wahrnehmung als für ihn gemeintes Signal des Nachrichtensprechers, dass die altbekannten Verfolger vor der Wohnungstür stünden. Dabei laufen die abduktiven Denkprozesse, die zu diesen Interpretationen führen, typischerweise automatisch und unbewusst ab und sind der Kontrolle des Betroffenen entzogen.

$\mathrm{Zu}$ einem wahnhaften Gedanken wird die „Hypothese“ aber erst dadurch, dass sie ein „asymmetrisches Inferenzprofil“ aufweist: Damit ist gemeint, dass der wahnhafte Gedanke zwar Auswirkungen auf viele andere Gedanken hat und durch Folgerungsbeziehung diese verändern kann, dass er aber gleichzeitig immun ist gegenüber der Veränderung durch andere Gedanken, insbesondere gegenüber neuen Gegen-Evidenzen. Das bedeutet, dass Betroffene an diesem Gedanken festhalten werden, ungeachtet aller Gegenanzeichen und Beteuerungen von Anderen. Gleichzeitig aber kann der wahnhafte Gedanke eine große Rolle für das übrige Überzeugungssystem spielen, sodass es zur Ausbildung ganzer „Wahngebäude“ kommt (,systematisierter Wahn“).

7 Wir gehen dabei nicht davon aus, dass alle Denkprozesse bewusst ablaufen müssen. Viele Prozesse, die gemeinhin als Denkprozesse eingeordnet werden, laufen automatisch und ohne kognitive Kontrolle ab. So verstehen wir z. B. mühelos und automatisch, dass der Sprecher mit dem Auto über die A46 gekommen ist, wenn er sagt: „Entschuldigung für meine Verspätung, auf der A46 war Stau." Tatsächlich erfordert ein solches Verständnis aber einige Schlussfolgerungen im Hintergrund (zusammen mit Hintergrundannahmen), die man gewöhnlich als Denkprozesse auffasst. In ähnlicher Weise dürften die meisten der abduktiven Hypothesenbildungen unbewusst und automatisch ablaufen. 
Die „Immunität gegenüber Revision“ ist also das zentrale Merkmal des Wahns. Da es sich dabei um eine ungewöhnliche Art handelt, mit Überzeugungen umzugehen, liegt die Vermutung nahe, dass auch auf dieser Ebene eine weitere Störung vorliegt. ${ }^{8}$ Damit müssen wir generell bei Wahn, ähnlich wie wir es schon oben für die Fremdbeeinflussung getan haben, zwischen zwei Ebenen unterscheiden: Zum einen gibt es eine Erfahrungsebene, auf der eine ungewöhnliche Wahrnehmung oder ein ungewöhnliches Gefühl auftritt, dass auf der Urteilsebene durch eine bestimmte Überzeugung erklärt wird. Wenn diese Überzeugung dann immun wird gegenüber Gegenevidenz, sprechen wir von einer wahnhaften Überzeugung.

Als ein Beispiel zur Illustration sei hier die Capgras-Illusion kurz vorgestellt. Patienten, die an dieser Illusion leiden, haben den Eindruck, dass eine ihnen nahe stehende Person (meist der Ehegatte oder die Eltern) durch eine andere Person ersetzt wurde, die aber genauso aussieht und sich genauso verhält. Dieser Eindruck entsteht wohl durch eine Störung in dem Gehirnareal, das der emotionalen Reaktion auf bekannte Gesichter zugeordnet werden kann (Ellis u. a. 1997; Hirstein/Ramachandran 1997). Daher können die Betroffenen zwar offenbar das bekannte Gesicht als solches erkennen und einer bestimmten Person zuordnen, aber die normale emotionale Reaktion bleibt aus. Es stellt sich ein Gefühl der Fremdheit gegenüber der bekannten Person ein. Um dieses Gefühl zu erklären, formulieren (manche) Patienten nun die Hypothese, dass die Person tatsächlich durch eine andere ersetzt wurde (Coltheart 2007). ${ }^{9}$ Dabei ist diese Hypothese zunächst nicht irrational, sondern sie bietet tatsächlich eine sehr gute Erklärung für die ungewöhnliche Erfahrung: Wenn es tatsächlich so wäre, dass der Ehegatte durch einen Betrüger ausgetauscht wurde, so wäre es zu erwarten, dass der Patient die

8 Tatsächlich ist dies letzten Endes aber eine empirische Frage. Natürlich gibt es viele Alltagsgedanken, die wir de facto nie ändern (etwa die Überzeugung, dass 1+1=2) und für die es schwer vorstellbar ist, wie eine Gegenevidenz überhaupt aussehen könnte. Ob wir allerdings wirklich wahnhaft an solchen Überzeugungen festhalten würden oder nicht, ist zunächst eine offene Frage. Mit Sicherheit ist es aber so, dass im Alltag die wahnhafte Überzeugung, dass $1+1=2$, nicht auffallen würde und daher die betreffende Person niemals als pathologisch eingestuft werden würde. Demnach bleibt aber auch offen, ob die zugrundeliegenden Denkprozesse bei Wahnpatienten tatsächlich gestört sind (was aufgrund der „Unglaublichkeit“ der wahnhaften Überzeugungen nahe liegt), oder ob der Denkprozess zwar normal, aber die in den Denkprozess eingehenden Prämissen aufgrund veränderter Wahrnehmung verzerrt sind.

9 Falls ein Patient diese Hypothese nicht aufstellt, spricht man von einer Illusion. Falls aber eine solche wahnhafte Überzeugung hinzutritt, wird die Capgras-Illusion zum Wahn. 
Person zwar erkennt, aber nicht die normale, automatische emotionale Reaktion erfährt. Insofern sich diese Hypothese dann als revisionsimmune Überzeugung etabliert, entsteht der Capgras-Wahn.

Vergleichbar sind nicht-pathologische Phänomene, zum Beispiel die Illusion eines „schwarzen Mannes“ auf einer nebligen Hochfläche - wobei sich der „schwarze Mann“ bei näherem Herantreten an das gewähnte Objekt als harmloser Busch entpuppt und dann auch korrekt als solcher wahrgenommen wird. Auch hier kommt es zu einer automatischen „Hypothesenbildung“; allerdings wird die Hypothese nicht zum Wahn, da die beim Herantreten neu gewonnenen Evidenzen korrekterweise zur Revision der Hypothese führen. In der Darstellungsmöglichkeit auch für solche normalpsychologischen (im Grenzbereich zur psychischen Störung angesiedelten) Phänomene liegt eine Stärke des Modells und der Frame-Darstellung. Auch ist diese Grundkonzeption therapeutisch hilfreich, denn sie erklärt beispielsweise therapeutische Effekte durch die kognitive Psychotherapie bei Wahnsymptomen, die letzten Endes auf eine „Neukalibrierung“ der automatischen Hypothesenbildung hinausläuft. Eine einfache Frame-Darstellung des Capgras-Wahns findet sich in Abb. 7.

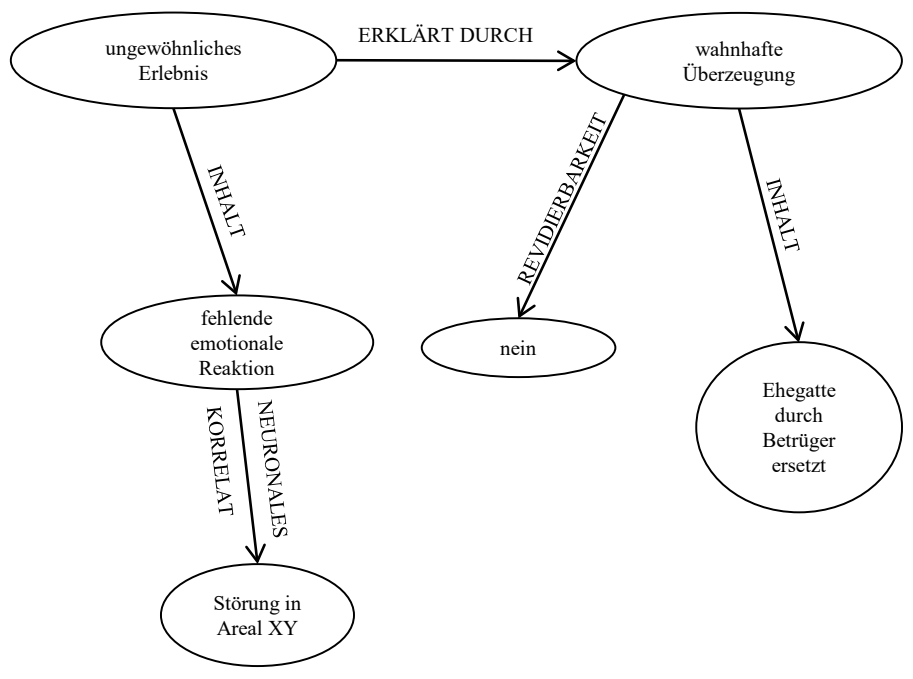

Abb. 7: Frame-Darstellung des Capgras-Wahns 
Offensichtlich sind aber einige Aspekte in dieser Darstellung noch nicht erfasst durch die rekursive Struktur von Frames lassen sich aber einzelne Aspekte, wie z. B. der Einfluss der wahnhaften Überzeugung zu anderen Überzeugungen, ergänzen. Ebenfalls müsste für eine umfassende Darstellung das neuronale Korrelat weit detaillierter dargestellt werden, was hier aber aus Platzgründen nicht geschehen soll. Wichtig ist an dieser Stelle nur zu zeigen, dass die Frame-Darstellung eine Erweiterung um diese Beschreibungsebene erlaubt. Ebenso kann, was in diesem Aufsatz allerdings nicht geleistet werden kann, auch die soziale Dimension, die einen entscheidenden Einfluss auf die Ausbildung von wahnhaften Überzeugungen und auf die dadurch entstehenden Einschränkungen der Betroffenen hat, aufgenommen werden.

Aus der (vereinfachten) Darstellung des sehr speziellen Capgras-Wahns ergibt sich eine allgemeine Darstellung von (monothematischem) Wahn (siehe Abb. 8).

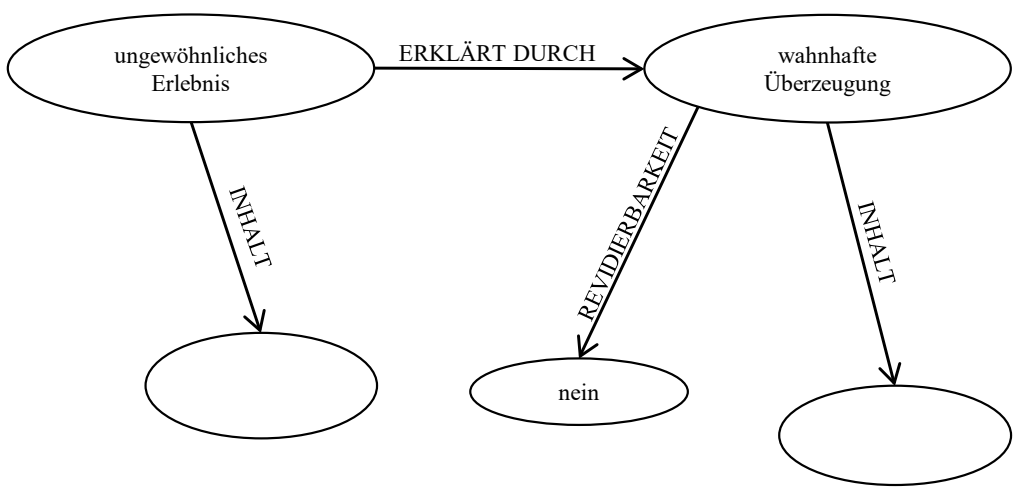

Abb. 8: Allgemeine Frame-Darstellung von monothematischem Wahn

In diesem Frame ist nur das Gerüst übernommen, das die Grundbestandteile von Wahnstörungen darstellt: Es gibt zwei Ebenen, nämlich die Ebene des ungewöhnlichen Erlebnisses und die Ebene der wahnhaften Überzeugung. Charakteristisch für Wahn sind nun zwei Elemente: Erstens erklärt die wahnhafte Überzeugung das ungewöhnliche Erlebnis, und zweitens ist die wahnhafte Überzeugung nicht revidierbar, also immun gegenüber Gegen-Evidenzen. Die Werte zu den beiden Inhalts-Attributen sind nicht angegeben, was anzeigt, dass zwar immer ein bestimmter Inhalt vorliegt, dass dieser Inhalt aber nicht entscheidend ist für Wahn. 
Allerdings können wir aufgrund des spezifischen Inhalts verschiedene Formen des Wahns unterscheiden, wie etwa Verfolgungswahn, Capgras-Wahn oder eben Fremdbeeinflussungswahn.

Wenn wir nun das Phänomen der Fremdbeeinflussung (siehe Abb. 5) kombinieren mit dem allgemeinen Frame für Wahn, so werden wir das UrheberschaftsGefühl als das ungewöhnliche Erlebnis identifizieren, dessen Inhalt ist, dass manche der eigenen Bewegungen als nicht selbst-verursacht erlebt werden. Die wahnhafte Überzeugung ist in diesem Fall das Urheberschafts-Urteil mit dem Inhalt, dass eine bestimmte andere Person Urheber dieser Bewegungen ist. Während in Abb. 5 das Urheberschafts-Gefühl lediglich als „cue“ für das Urteil angeführt wird, wird durch die Kombination mit dem Wahn-Frame eine neue Beziehung zwischen beiden deutlich, nämlich dass das Urheberschafts-Urteil auch das Urheberschafts-Gefühl erklärt. ${ }^{10}$ Auf diese Weise kann nun gleichzeitig erfasst werden, wie nicht-pathologische Urheberschafts-Urteile zustande kommen und wie wahnhafte Fremdbeeinflussungs-Überzeugungen entstehen. Die spezifischen Ursachen des Fremdbeeinflussungs-Wahns können nun in verschiedenen Bereichen des Geistes ausgemacht werden, wobei in diesem Fall sowohl motorische als auch Wahrnehmungsstörungen infrage kommen. So kann auch die (mögliche) komplexe Auswirkung von lokal begrenzten Störungen etwa im Bereich der Propriozeption auf andere Bereiche wie dem Selbst-Bewusstsein nachvollzogen werden.

Darüber hinaus können verschiedene Aspekte eines Phänomens, nämlich der Fremdbeeinflussung, übersichtlich dargestellt werden: Alle oben genannten unterschiedlichen Klassifikationen finden sich in der Frame-Darstellung wieder (motorische Störung, Ich-Störung, Wahn) und können zueinander in Beziehung gesetzt werden. Dadurch entsteht nicht nur ein umfassenderes Verständnis der Phänomene, sondern es ergibt sich auch eine neue Möglichkeit der Klassifikation, die sich nicht nur an den gestörten „Bereichen“ orientiert, sondern an den unterschiedlichen Faktoren, die bei der Entstehung eine Rolle spielen. So wird der systematische Vergleich zwischen Symptomen möglich, die durch die bisherige Klassifizierung als völlig verschiedene Bereiche betreffend eingeordnet wurden und

${ }^{10}$ Das gilt allerdings nur für den Wahn: Im Alltag kann, wie oben am Beispiel mit der Vase gezeigt, durchaus das Gefühl vom Urteil abweichen, ohne dass das Urteil irgendeine Erklärung für das Gefühl bereithalten würde. 
daher auch nicht verglichen wurden (das gilt auch für die Möglichkeit, verschiedene Ursachen für die lokalen Störungen miteinzubeziehen, etwa Drogenmissbrauch vs. genetische Faktoren, die in den bisherigen Klassifikationssystemen zu völlig unterschiedlichen Einordnungen führen).

Die hier vorgestellte Theorie der Wahnphänomene ist ein Modell, das sich empirisch überprüfen lässt. Im Gegensatz zu dem ganz ähnlichen Modell der abduktiven Inferenz von Coltheart et al. (2010) ist es allerdings offener für Störungsverursachungen, die nicht nur eine fehlerhafte Wahrnehmung oder eine Komparator-Fehlfunktion annehmen, und es erlaubt, neuere Erkenntnisse über die Pathophysiologie des Wahns zu nutzen, um Verfeinerungen des Modells vorzunehmen. Hier sind vor allem Modelle einer vermehrten Neigung zu voreiligem Schlussfolgern sowie Modelle einer exzessiven Selbstreferenz als Ergänzungen und Alternativen zu nennen, die in künftigen Erweiterungen der Frame-Darstellungen des Wahns zu berücksichtigen sind (Braun/Suffren 2011). Dafür wäre eine systematische Literaturübersicht zu empirischen Untersuchungen zur Pathophysiologie des Wahns und der Halluzinationen wünschenswert, die als Grundlage für eine empirisch fundierte Überarbeitung des Frames herangezogen werden könnte. Umfassende Modelle des Wahns müssen auch die bereits erwähnten Aspekte der neurogenetischen Faktoren und der soziokulturellen Faktoren, die bei der individuellen Ausprägung der Wahninhalte eine wichtige Rolle spielen (Cannon/Kramer 2012), mit einbeziehen. Hier kommen auch affektive Faktoren (Garety u. a. 2013) und Umweltfaktoren ins Spiel, wobei letztere entweder über epigenetische Regulationsschritte wie (symptomspezifische?) DNA-Methylierungen (Liu u. a. 2013) oder über die urbane Umgebung und deren psychologische Auswirkungen (Ellett/Freeman/Garety 2008) das Auftreten und/oder das klinische Ausprägungsbild psychotischer Störungen modifizieren.

\section{Zusammenfassung}

Am Beispiel des Symptoms der Fremdbeeinflussung haben wir eine komplexe Frame-Darstellung entwickelt, die ganz unterschiedlichen Aspekten dieses Phänomens gerecht wird. Diese Darstellung erlaubt ein wesentlich detaillierteres Verständnis des komplexen Phänomens und damit auch eine Klassifizierung, die bisher nicht beachtete Parallelen zu anderen Symptomen aufzeigen kann. Dabei 
orientiert sich die Klassifizierung, die auf der Grundlage von Frame-Darstellungen erfolgt, nicht mehr am Bereich, der angeblich gestört ist, sondern an den einzelnen Faktoren, die für die Entstehung des Symptoms eine Rolle spielen. Auf diese Weise werden auch die komplexen Zusammenhänge zwischen Bereichen, die traditionell als getrennt voneinander behandelt werden (z. B. Motorik und selbstbewusste Überzeugungen), sichtbar.

Über die Vorteile für die Klassifikation psychischer Störungen hinaus ergeben sich wertvolle Erkenntnisse für die Funktionsweise des menschlichen Geistes im Allgemeinen. Generell gilt, dass wenn bestimmte Funktionen gestört sind, wir dann oft besser die Funktion verstehen. Auch in diesem Fall hilft uns das Symptom der Fremdbeeinflussung, die komplexen Zusammenhänge zwischen verschiedenen funktionalen Einheiten („Bereichen“) des menschlichen Geistes zu verstehen. Auf diese Art und Weise können wir nicht nur eine spezifische Theorie der Bildung von Urheberschafts-Urteilen im Alltag entwickeln, wir können auch den allgemeinen Zusammenhang zwischen dem motorischen Bereich und dem Bereich der selbstbewussten Urteile nachvollziehen.

Die Frame-Analyse von psychischen Störungen trägt damit zu einem umfassenden Verständnis der „Architektur des Geistes“ bei. Dieses Verständnis zielt vor allem auf die detaillierte Darstellung der Zusammenhänge der unterschiedlichen Einheiten ab, wohingegen „radikale“ Ansätze entweder systematische Interaktionen (die über den Informationsaustausch hinausgehen) zwischen Bereichen ausschließen (so in einigen Aspekten der älteren Modularitätsthesen; Übersicht bei Zielasek/Gaebel 2008) oder jeglicher Aufteilung des Geistes in funktionell abgrenzbare Bereiche den Sinn absprechen. ${ }^{11}$

Das Ziel einer Frame-Darstellung aller psychischen Symptome ist daher nicht nur eine vollständige Darstellung aller Symptome, sondern auch die Erstellung eines Personen-Frames, der alle Aspekte einer gesunden wie einer kranken Person erfasst, und in dem die komplexen Auswirkungen unterschiedlicher lokal begrenzter oder systemisch einwirkender Störungsfaktoren nachvollzogen werden können. Selbstverständlich ist ein solch umfassender Personen-Frame, der auch die gesamte Architektur des Geistes enthalten würde, nicht nur enorm komplex, sondern auch ein Ziel, das in weiter Ferne liegt. Die Arbeit an diesem Ziel kann

\footnotetext{
${ }^{11}$ Für eine Diskussion solcher Thesen siehe auch Weber/Vosgerau (2012).
} 
aufgrund der rekursiven Struktur von Frames allerdings auch in kleinen Schritten erfolgen: Egal auf welcher Ebene wir anfangen und mit welchen Detailgrad wir beginnen, wir können die Frame-Darstellung jederzeit erweitern durch weitere Details und durch „Brücken“ auf andere Beschreibungsebenen. ${ }^{12}$

\section{Literatur}

American Psychiatric Association (ed.) (2013): Diagnostic and Statistic Manual of Mental Disorders, Fifth Edition (DSM-5). Arlington: American Psychiatric Association.

Barsalou, L. W. (1992): Frames, Concepts and Conceptual Fields. In: A. Lehrer / E. Feder Kittay (eds.): Frames, Fields and Contrasts. Hillsdale / Hove / London: Erlbaum, 21-74.

Beckermann, A. (2010): Die Rede von dem Ich und dem Selbst. Sprachwidrig und philosophisch höchst problematisch. In: K. Crone / R. Schnepf / J. Stolzenberg (Hrsg.): Über die Seele. Berlin: Suhrkamp, 458-473.

Braun, C. M. J. / Suffren, S. (2011): A general neuropsychological model of delusion. In: Cognitive Neuropsychiatry 16, 1-39.

Cannon, B. J. / Kramer, L. M. (2012): Delusion Content across the 20th Century in an American Psychiatric Hospital. In: International Journal of Social Psychiatry 58 (3), 323-27. doi:10.1177/0020764010396413.

Carruthers, G. (2012): The case for the comparator model as an explanation of the sense of agency and its breakdowns. In: Beyond the Comparator Model 21 (1), 30-45. doi:10.1016/j.concog.2010.08.005.

Coltheart, M. (2007): Cognitive Neuropsychiatry and Delusional Belief. In: Quarterly Journal of Experimental Psychology (2006) 60 (8), 1041-1062.

Coltheart, M. / Menzies, P. / Sutton, J. (2010): Abductive Inference and Delusional Belief. In: Cognitive Neuropsychiatry 15 (1-3), 261-287. doi:10.1080/ 13546800903439120 .

\footnotetext{
12 Der Beitrag ist im Teilprojekt B06 des DFG-geförderten Sonderforschungsbereichs 991 „The Structure of Representations in Language, Cognition, and Science" entstanden. Wir danken der DFG für die großzügige Förderung.
} 
Davies, M. / Coltheart, M. / Langdon, R. / Breen, N. (2001): Monothematic Delusions: Towards a Two-Factor Account. In: Philosophy, Psychiatry, and Psychology 8 (2-3), 133-158.

Ellett, L. / Freeman, D. / Garety, P. A. (2008): The Psychological Effect of an Urban Environment on Individuals with Persecutory Delusions: The Camberwell Walk Stud“. In: Schizophrenia Research 99 (1-3), 77-84. doi:10.1016/ j.schres.2007.10.027.

Ellis, H. D. / Young, A. W. / Quayle, A. H. / De Pauw, K. W. (1997): Reduced autonomic responses to faces in Capgras delusion. In: Proceedings of the Royal Society of London: Biological Sciences 264 (1384): 1085-1092.

Enoch, D. (1991): Delusional Jealousy and Awareness of Reality. In: The British Journal of Psychiatry 159 (14), 52-56.

Frith, C. D. (1992): The Cognitive Neuropsychology of Schizophrenia. Hillsdale: Erlbaum.

Firth, C. D. (2005): The self in action: Lessons from delusions of control. In: Consciousness and Cognition 14 (4), 752-770.

Frith, C. D. / Blakemore, S.-J. / Wolpert, D. M. (2000): Abnormalities in the awareness and control of action. In: Philosophical Transactions of the Royal Society of London B 355 (1404), 1771-1788.

Gaebel, W. / Zielasek, J. (2009): Future Classification of Psychotic Disorders. In: European Archives of Psychiatry and Clinical Neuroscience 259 (2), 213-218.

Gaebel, W. / Zielasek, J. (2011): Experience with ICD-10 in Europe and Scientific Evidence for New Aspects of ICD-11 from German-Language Publications. In: European Psychiatry 26 (Suppl. 2), 6-10.

Gaebel, W. / Zielasek, J. / Cleveland, H. R. (2012): Classifying Psychosis - Challenges and Opportunities. In: International Review of Psychiatry 24 (6), 538-548. doi:10.3109/09540261.2012.737313.

Garety, P. A. / Gittins, M. / Jolley, S. / Bebbington, P. / Dunn, G. / Kuipers, E. / Fowler, D. / Freeman, D. (2013): Differences in Cognitive and Emotional Processes Between Persecutory and Grandiose Delusions. In: Schizophrenia Bulletin 39 (3), 629-639. doi:10.1093/schbul/sbs059. 
Hirstein, W. / Ramachandran, V. S. (1997): Capgras syndrome: A novel probe for understanding the neural representation of the identity and familiarity of persons. In: Proceedings of the Royal Society of London: Biological Sciences 264 (1380), 437-444.

Lindner, A. / Thier, P. / Kircher, T. T. J. / Haarmeier, T. / Leube, D. T. (2005): Disorders of Agency in Schizophrenia Correlate with an Inability to Compensate for the Sensory Consequences of Actions. In: Current Biology 15 (12), 1119-1124. doi:10.1016/j.cub.2005.05.049.

Liu, J. / Chen, J. / Ehrlich, S. / Walton, E. / White, T. / Perrone-Bizzozero, N. / Bustillo, J. / Turner, J. A. / Calhoun, V. D. (2013): Methylation Patterns in Whole Blood Correlate With Symptoms in Schizophrenia Patients. In: Schizophrenia Bulletin, Juni. doi:10.1093/schbul/sbt080. http://schizophreniabulletin.oxfordjournals.org/cgi/doi/10.1093/schbul/sbt080.

McKay, R. (2012): Delusional Inference. In: Mind \& Language 27 (3), 330-355. doi:10.1111/j.1468-0017.2012.01447.x.

Moritz, S. / Woodward, T. S. (2005): Jumping to conclusions in delusional and non-delusional schizophrenic patients. In: British Journal of Clinical Psychology 44, 193-207.

Petersen, W. (2007): Representation of Concepts as Frames. In: The Baltic International Yearbook of Cognition, Logic and Communication 2, 151-170.

Shergill, S. S. / Samson, G. / Bays, P. M. / Frith, C. D. / Wolpert, D. (2005): Evidence for Sensory Prediction Deficits in Schizophrenia. In: American Journal of Psychiatry 162 (12), 2384-2386. doi:10.1176/appi.ajp.162.12.2384.

Soom, P. / Vosgerau, G. (2015): A Functionalist Approach to the Concept of ,Delusion'. In: Journal für Philosophie und Psychiatrie, www.jfpp.org/113.html.

Sperry, R. W. (1950): Neural Basis of the Spontaneous Optokinetic Response Produced by Visual Inversion. In: Journal of Comparative and Physiological Psychology 43, 482-489.

Synofzik, M. / Thier, P. / Leube, D. T. / Schlotterbeck, P. / Lindner, A. (2010): Misattributions of agency in schizophrenia are based on imprecise predictions about the sensory consequences of one's actions. In: Brain 133, 262-271. doi:10.1093/brain/awp291.

Synofzik, M. / Vosgerau, G. / Lindner, A. (2009): Me or not me - An optimal integration of agency cues? In: Consciousness and Cognition 18, 1065-1068. 
Synofzik, M. / Vosgerau, G. / Newen, A. (2008a): Beyond the comparator model: A multifactorial two-step account of agency. In: Consciousness and Cognition 17, 219-239.

Synofzik, M. / Vosgerau, G. / Newen, A. (2008b): I move, therefore I am: A new theoretical framework to investigate agency and ownership. In: Consciousness and Cognition 17, 411-424.

Synofzik, M. / Voss, M. (2010): Disturbances of the sense of agency in schizophrenia. In: Balconi, M. (ed.): Neuropsychology of the sense of agency. New York: Springer, 145-156.

Von Holst, E. / Mittelstaedt, H. (1950): Das Reafferenzprinzip. In: Die Naturwissenschaften 20, 464-476.

Vosgerau, G. (2009a): Mental Representation and Self-Consciousness. From Basic Self-Representation to Self-Related Cognition. Paderborn: mentis.

Vosgerau, G. (2009b): Stufen des Selbstbewusstseins: Eine Analyse von Ich-Gedanken. In: Grazer Philosophische Studien 78, 101-130.

Vosgerau, G. (2009c): Die Stufentheorie des Selbstbewusstseins und ihre Implikationen für das Verständnis psychiatrischer Störungen. In: Journal für Philosophie und Psychiatrie 2 (2). http://www.jfpp.org/jfpp-2-2009-02.html.

Vosgerau, G. / Synofzik, M. (2012): Weighting models and weighting factors. Consciousness and Cognition 21, 55-58.

Weber, A. / Vosgerau, G. (2012): Grounding Action Representations. In: Review of Philosophy and Psychology 3, 53-69.

Woodward, T. S. / Moritz, S. / Cuttler, C. / Whitman, J. C. (2006): The Contribution of a Cognitive Bias Against Disconfirmatory Evidence (BADE) to Delusions in Schizophrenia. In: Journal of Clinical and Experimental Neuropsychology 28, 605-17.

World Health Organization (1992): The ICD-10 Classification of Mental and Behavioural Disorders. Geneva: World Health Organization.

Zielasek, J. / Gaebel, W. (2008): Modern Modularity and the Road towards a Modular Psychiatry. In: European Archives of Psychiatry and Clinical Neuroscience 258 (Suppl. 5), 60-65. 
